(C) 1998 International Press

Adv. Theor. Math. Phys. 2 (1998) 853-871

\title{
Classification of Reflexive Polyhedra in Three Dimensions
}

\author{
Maximilian Kreuzer ${ }^{\mathrm{a}}$, Harald Skarke ${ }^{\mathrm{b}}$ \\ ${ }^{a}$ Institut für Theoretische Physik \\ Technische Universität Wien \\ Wiedner Hauptstraße 8-10 \\ A-1040 Wien, AUSTRIA \\ maximilian.kreuzer@tuwien.ac.at \\ ${ }^{b}$ Theory Group \\ Department of Physics \\ University of Texas \\ Austin, TX 78712, USA \\ skarke@zerbina.ph.utexas.edu
}

\begin{abstract}
We present the last missing details of our algorithm for the classification of reflexive polyhedra in arbitrary dimensions. We also present the results of an application of this algorithm to the case of three dimensional reflexive polyhedra. We get 4319 such polyhedra that give rise to K3 surfaces embedded in toric varieties. 16 of these contain all others as subpolyhedra. The 4319 polyhedra form a single connected web if we define two polyhedra to be connected if one of them contains the other.
\end{abstract}

e-print archive: http://xxx.lanl.gov/abs/hep-th/9805190 


\section{INTRODUCTION}

\subsection{MOTIVATION}

When it was first realized [1] that manifolds of trivial canonical class play an important role in string compactifications, only very few manifolds of this type were known. This situation changed with the first construction of large classes of Calabi-Yau manifolds as hypersurfaces in weighted projective spaces [2]. As these varieties are generically singular, it was not clear from a mathematical point of view what their Hodge numbers should be, but in string theory the corresponding numbers of generations and antigenerations could be calculated by using orbifold techniques [3]. These 'physicists' Hodge numbers' showed a remarkable property that became known as mirror symmetry: to almost every manifold with a certain pair of Hodge numbers there existed one or more other manifolds with the Hodge numbers exchanged. Soon an explicit construction applying to a subset of these spaces was found [4]. But when a complete classification of all such varieties was available $[5,6]$ it became clear that mirror symmetry is not realized at the level of hypersurfaces in weighted projective spaces.

Indeed, a far more natural setup for the discussion of mirror symmetry is given in terms of toric geometry and in particular by hypersurfaces in toric varieties that can by described by so-called reflexive polyhedra [7]. This construction not only implies manifest mirror symmetry (at the level of Hodge number exchange), but also explains how the weighted projective spaces have to be desingularised (blown up) in order to allow smooth hypersurfaces with the Hodge numbers that have been assigned to them through the orbifold construction.

It is often possible to obtain a Calabi-Yau manifold from another CalabiYau manifold by first blowing down some divisors, thereby creating a singular variety, and then resolving the singularity by changing the complex structure. This raises the question of whether all Calabi-Yau manifolds might be connected (directly or indirectly) by processes of this sort [8], which lead to important non-perturbative effects in string theory $[9,10]$. It has been shown $[11,12]$ that all Calabi-Yau threefolds that are hypersurfaces in weighted projected spaces belong to a 'web' of this type. This web could not be formed from hypersurfaces in weighted projective spaces alone, but required, once again, a generalization to toric hypersurfaces.

It should be noted that almost all examples of manifolds of trivial canonical bundle occurring in the physics literature are hypersurfaces (or, in a few 
cases, complete intersections) in toric varieties. Therefore a classification of toric varieties that admit smooth three-dimensional hypersurfaces of vanishing first Chern class is highly desirable. Such a classification amounts to the classification of four-dimensional reflexive polyhedra. While this classification problem is rather easy in two dimensions (there are 16 well known reflexive polygons), only recently an algorithm for approaching this problem in higher dimensions was found $[13,14]$. In the present work we fill in the last missing technical details of our algorithm and apply it to the classification of three dimensional reflexive polyhedra. At first sight this might seem to be rather useless, since any hypersurface resulting from this construction is going to be a K3 surface and all K3 surfaces are known to be isomorphic with respect to their differential structures. This is misleading, however, since in the context of string dualities algebraic properties become important, and these algebraic properties are conveniently encoded in the structures of the polyhedra. When we consider F-theory or IIA duals to heterotic string compactifications, we usually consider Calabi-Yau threefolds or fourfolds that are K3 fibrations where the K3s themselves are elliptically fibered. Then the fibration structures manifest themselves as nestings of the respective polyhedra $[15,16,17]$, and even the enhanced gauge groups can be read off from the toric diagrams $[15,18,19]$. Thus, the toric diagrams contain far more information than just which differential type of manifold we are dealing with.

In the remainder of the introduction we will give some definitions necessary in the rest of the paper. In section 2 we give a rough outline of the strategy that we used for the classification and present our results. In section 3 we explain our algorithm in more detail, starting with a summary of the results of refs. $[13,14]$ and then proceeding to more detailed descriptions of various ideas that were relevant in the course of implementing our algorithm.

\subsection{BASIC DEFINITIONS}

A polytope in $\mathbb{R}^{n}$ may be defined alternatively as the convex hull of finitely many points or as an intersection of finitely many half spaces that is bounded. In the mathematics literature a polyhedron is also an intersection of finitely many half spaces, but not necessarily bounded [20]. We will, however, always mean 'polytope' even when we write 'polyhedron'. More particularly, most of the polyhedra that we consider will be polytopes with $\mathbf{0}$ (the origin of $\mathbb{R}^{n}$ ) in the interior. We will denote this property as the 'interior point property' or 'IP property'. Given a polytope $\Delta$ in a vector space $M_{\mathbb{R}} \simeq \mathbb{R}^{n}$ with the 
IP property, we may define the dual (or polar) polytope $\Delta^{*} \subset N_{\mathbb{R}}=M_{\mathbb{R}}^{*}$ as

$$
\Delta^{*}=\left\{y \in N_{\mathbb{R}}: \quad\langle y, x\rangle \geq-1 \quad \forall x \in M_{\mathbb{R}}\right\},
$$

where $\langle y, x\rangle$ is the duality pairing between $y \in N_{\mathbb{R}}$ and $x \in M_{\mathbb{R}}$. Because of the convexity of $\Delta,\left(\Delta^{*}\right)^{*}=\Delta$.

Given a dual pair of polytopes such that $\Delta$ has $n_{V}$ vertices and $n_{F}$ facets (a facet being a codimension 1 face), the dual polytope has $n_{V}$ facets and $n_{F}$ vertices. We may then define the vertex pairing matrix (VPM) $X$ as the $n_{F} \times n_{V}$ matrix whose entries are $X_{i j}=\left\langle\bar{V}_{i}, V_{j}\right\rangle$, where $\bar{V}_{i}$ and $V_{j}$ are the vertices of $\Delta^{*}$ and $\Delta$, respectively. $X_{i j}$ will be -1 whenever $V_{j}$ lies on the i'th facet. Note that $X$ is independent of the choice of a dual pair of bases in $N_{\mathbb{R}}$ and $M_{\mathbb{R}}$ but depends on the orderings of the vertices.

Given a lattice $M$, a lattice (or integer) polyhedron is a polyhedron on the real extension $M_{\mathbb{R}}$ of $M$ whose vertices lie in $M$. A lattice polyhedron $\Delta \subset M_{\mathbb{R}}$ is called reflexive if its dual $\Delta^{*} \subset N_{\mathbb{R}}$ is a lattice polyhedron w.r.t. the lattice $N$ dual to $M$. In this case the elements of the vertex pairing matrix $X$ are integer. Note that, in turn, integer $X$ implies that there is a (finite number of) lattice(s) with respect to which the polyhedron is reflexive. The coarsest such lattice is generated by the vertices of $\Delta$ and is a sublattice of the finest lattice of this type, which is dual to the lattice generated by the vertices of $\Delta^{*}$.

The lattice points of a reflexive polyhedron $\Delta$ encode the monomials occurring in the description of the hypersurface in a variety whose fan is determined by a triangulation of the dual polyhedron $\Delta^{*}$. For details of what a fan is and how it determines a toric variety, it is best to look up a standard textbook [21, 22].

If a polyhedron $\Delta_{1}$ contains a polyhedron $\Delta_{2}$, then the definition of duality implies $\Delta_{1}^{*} \subset \Delta_{2}^{*}$. Therefore the variety determined by the fan over $\Delta_{1}^{*}$ may be obtained from the variety determined by the fan over $\Delta_{2}^{*}$ by blowing down one or several divisors. If we perform this blow-down while keeping the same monomials (those determined by $\Delta_{2}$ ), we obtain a generically singular hypersurface. This hypersurface can be desingularised by varying the complex structure in such a way that we now allow monomials determined by $\Delta_{1}$. Thus the classes of Calabi-Yau hypersurfaces determined by polyhedra $\Delta_{1}$ and $\Delta_{2}$, respectively, can be said to be connected whenever $\Delta_{1}$ contains $\Delta_{2}$ or vice versa. More generally, if there is a chain of polyhedra $\Delta_{i}$ such that $\Delta_{i}$ and $\Delta_{i+1}$ are connected in the sense defined above, we call the hypersurfaces corresponding to any two elements of the chain connected. 


\section{STRATEGY AND RESULTS}

Our approach to the classification of all reflexive polyhedra starts with the construction of a set of maximal objects that contain all reflexive polyhedra as subsets. Finding such a set in principle solves the classification problem, but in practice the 'trivial' second step of enumerating all reflexive subpolyhedra may be quite tricky or even impossible because of constraints of space and time. In section 3 we describe in some detail which algorithms we used to complete the classification for the 3-dimensional case. But first we present a road map that shows how the pieces fit together.

\begin{tabular}{|c|c|c|c|c|c|c|c|c|c|c|c|c|}
\hline$d^{(i)}$ & 4 & 5 & 6 & 6 & 77 & 8 & 99 & 10 & 12 & 32 & $\overline{422}$ & $\overline{33}$ \\
\hline$w_{1}^{(i)}$ & 1 & 1 & 1 & 1 & 1 & 1 & 1 & 1 & 1 & 10 & 10 & 11 \\
\hline$w_{2}^{(i)}$ & 1 & 1 & 1 & 1 & 1 & 1 & 1 & 1 & 1 & 10 & 10 & 10 \\
\hline$w_{3}^{(i)}$ & 1 & 1 & 2 & 1 & 2 & 2 & 3 & 3 & 4 & 10 & 20 & 10 \\
\hline $\begin{array}{l}w_{4}^{(i)} \\
w_{5}^{(i)} \\
w_{6}^{(i)}\end{array}$ & 1 & 2 & 2 & 3 & 3 & 4 & 4 & 5 & 6 & $\begin{array}{ll}0 & 1 \\
0 & 1\end{array}$ & $\begin{array}{ll}0 & 1 \\
0 & 1\end{array}$ & \\
\hline Points & 35,19 & $\overline{34}$ & $\overline{30}$ & 39 & 31 & 35 & 33 & $\overline{c 36}$ & $\bar{~} 39$ & 30 & 27 & $\bar{~} 30$ \\
\hline
\end{tabular}

\begin{tabular}{||c||cc|c|c||cc|c||}
\hline \hline$d^{(i)}$ & 3 & 4 & 4 & 4 & 2 & 2 & 2 \\
\hline$w_{1}^{(i)}$ & 1 & 2 & 2 & 2 & 1 & 0 & 0 \\
$w_{2}^{(i)}$ & 1 & 0 & 1 & 0 & 1 & 0 & 0 \\
$w_{3}^{(i)}$ & 1 & 0 & 1 & 0 & 0 & 1 & 0 \\
$w_{4}^{(i)}$ & 0 & 1 & 0 & 1 & 0 & 1 & 0 \\
$w_{5}^{(i)}$ & 0 & 1 & 0 & 1 & 0 & 1 & 0 \\
$w_{6}^{(i)}$ & & & & 0 & 0 & 1 \\
\hline \hline \hline Points & 31 & 35 & 27 \\
\hline \hline
\end{tabular}

Table 1: The 9 single and the 6 combined weight systems defining the polytopes containing all others, and the respective numbers of points.

Certain lattice polyhedra $\Delta$ can be described in the following simple way: Take the intersection of all positive half spaces $x_{i} \geq 0$ with the set of integer solutions to a linear equation $\sum q_{i} x_{i}=1$ with positive rational coefficients $q_{i}>0$, and define $\Delta$ to be the convex hull of these points. If $\sum q_{i}=1$ then $\Delta$ has at most one interior lattice point, namely the point 1 with all coordinates $x_{i}=1$. Our approach is based upon two crucial facts [13, 14]:

1. In any dimension $n$ there is only a finite number of (single) weight systems 
$\left(q_{i}\right)$ with $\sum q_{i}=1$ such that $\mathbf{1}$ is in the interior of the corresponding polyhedra. (By definition one interior lattice point is necessary for reflexivity; in $n \leq 4$ dimensions it is also sufficient for polyhedra of this type [14].)

2. Each reflexive polyhedron is contained in an object that is slightly more general: We may have to embed it into $\mathbb{Z}^{k}$ with codimension $k-n>1$ using sets of solutions to $k-n$ equations of the type $\sum q_{j}^{(i)} x_{j}=1$. There is a finite number of possible types of such combined weight systems, which consist of $k-n$ single weight systems which are extended by zeros (see section 3.1 or [13]).

The last entry in table 1, for example, corresponds to a cube embedded in $\mathbb{R}^{6}$ by

$$
x_{1}+x_{2}=2, \quad x_{3}+x_{4}=2, \quad x_{5}+x_{6}=2 \quad \text { and } \quad x_{i} \geq 0 \quad \forall i .
$$

In the three examples of table 1 with bi-degrees $\left(d^{(1)}, d^{(2)}\right)$ equal to $(3,3)$, $(3,4)$ and $(4,4)$, respectively, the coordinate $x_{1}$ enters both equations.

\begin{tabular}{||c||c|c|c|c|c|c|c|c|c|c||}
\hline \hline Points & 5 & 6 & 7 & 8 & 9 & 10 & 11 & 12 & 13 & 14 \\
\hline Multiplicity & 1 & 7 & 23 & 54 & 135 & 207 & 314 & 373 & 416 & 413 \\
\hline \hline Points & 15 & 16 & 17 & 18 & 19 & 20 & 21 & 22 & 23 & 24 \\
\hline Multiplicity & 413 & 348 & 334 & 274 & 234 & 179 & 151 & 117 & 87 & 66 \\
\hline \hline \hline Points & 25 & 26 & 27 & 28 & 29 & 30 & 31 & 32 & 33 & 34 \\
\hline Multiplicity & 40 & 42 & 27 & 18 & 8 & 13 & 9 & 4 & 2 & 2 \\
\hline \hline \hline Points & 35 & 36 & 39 & & & & & & & \\
\hline Multiplicity & 5 & 1 & 2 & & & & & & & \\
\hline \hline
\end{tabular}

Table 2: Multiplicities of point numbers for the 4319 reflexive polytopes.

\begin{tabular}{||c||c|c|c|c|c|c|c|c|c||}
\hline \hline Picard number & 1 & 2 & 3 & 4 & 5 & 6 & 7 & 8 & 9 \\
\hline Multiplicity & 2 & 9 & 25 & 58 & 101 & 165 & 254 & 372 & 489 \\
\hline \hline Picard number & 10 & 11 & 12 & 13 & 14 & 15 & 16 & 17 & 18 \\
\hline Multiplicity & 574 & 578 & 521 & 451 & 350 & 204 & 112 & 40 & 12 \\
\hline \hline Picard number & 19 & & & & & & & & \\
\hline Multiplicity & 2 & & & & & & & & \\
\hline \hline
\end{tabular}

Table 3: Multiplicities of Picard numbers for the 4319 reflexive polytopes.

According to [14, 23], there are 58 single and 21 combined weight systems relevant to our classification scheme. Our first new result is that these 
numbers may still be reduced: It turns out that all 3-dimensional reflexive polyhedra are contained in the 15 polyhedra that are defined by the weight systems in table 1 (cf. section 3.2). There is, however, a subtle point: This statement is true only if we also admit sublattices of the lattice that is defined by integer $x_{i}$. Indeed, it turns out that there is one polytope that we would miss if we ignored sublattices: It is a $\mathbb{Z}_{2}$ quotient with 19 lattice points of the simplex

$$
\left\{\left(x_{1}, x_{2}, x_{3}\right): x_{i} \geq-1 \wedge x_{1}+x_{2}+x_{3} \leq 1\right\}
$$

with 35 lattice points that is defined by the single weight system with degree 4 (the coordinates have been shifted by 1 such that the interior lattice point is at the origin). To obtain the 16 th polytope that is not a subpolytope of any other, we can restrict our lattice, for example, to $x_{1}+x_{2} \equiv 0 \bmod 2$, which keeps 19 of the 35 points, including all vertices and $\mathbf{0}$. In other words, we take out every other lattice plane parallel to a fixed set of two non-intersecting edges. Because of the full permutation symmetry of the 4 vertices there are 3 different choices of such a plane, which lead to the same polytope up to lattice automorphisms. In terms of toric geometry, we have the following interpretation: The simplex with 35 lattice points is the Newton polytope of the quartic hypersurface in $\mathbb{P}^{3}$. The $\mathbb{Z}_{2}$ quotient of lattices corresponds to a $\mathbb{Z}_{2}$ quotient of toric varieties. $\mathbb{P}^{3} / \mathbb{Z}_{2}$ has singularities at the fixed lines $\left(\mathbb{P}^{1} \mathrm{~s}\right)$ $z_{1}=z_{2}=0$ and $z_{3}=z_{4}=0$ of the $\mathbb{Z}_{2}$ action (the $z_{i}$ being the homogeneous coordinates of the $\mathbb{P}^{3}$ ). These singular lines must be blown up to obtain a smooth toric variety in which we have a K3 hypersurface whose Newton polytope is the simplex with 19 lattice points.

The fact that every polyhedron $\Delta$ is contained in at least one of the 16 objects we just discussed implies that the dual $\Delta^{*}$ contains one of the duals of these 16 polytopes. Therefore, the fan of any toric ambient variety determined by a maximal triangulation of a reflexive polyhedron is a refinement of one of the corresponding 16 fans. In other words, any such toric ambient variety is given by the blow-up of one of the following 16 spaces (cf. table 1):

$-\mathbb{P}^{3}$

$-\mathbb{P}^{3} / \mathbb{Z}_{2}$,

- 8 different weighted projective spaces $\mathbb{P}_{\left(q_{1}, q_{2}, q_{3}\right)}^{2}$,

$-\mathbb{P}^{2} \times \mathbb{P}^{1}$,

$-\mathbb{P}_{(1,1,2)}^{2} \times \mathbb{P}^{1}$

-3 further double weighted spaces, and

$-\mathbb{P}^{1} \times \mathbb{P}^{1} \times \mathbb{P}^{1}$.

Each of the three spaces with 'overlapping weights' allows two distinct bundle structures: The first one can be interpreted as a $\mathbb{P}^{2}$ bundle in two distinct 
ways, the second one as a $\mathbb{P}^{2}$ bundle or a $\mathbb{P}_{(1,1,2)}^{2}$ bundle, and the third one can be interpreted as a $\mathbb{P}_{(1,1,2)}^{2}$ bundle in two distinct ways. In each case the base space is $\mathbb{P}^{1}$.

In order to enumerate all 3-dimensional reflexive polytopes we thus had to construct all lattice subpolytopes $\Delta$ of the 15 objects defined by the weight systems in table 1 such that $\Delta$ is reflexive on some lattice. We first found 6202 inequivalent subpolytopes with integral VPM (polytopes that are reflexive on some lattice; cf. section 1.2), 4318 of which are reflexive on the original lattice. Then we computed the resulting 4075 inequivalent VPMs (to obtain these numbers we defined and computed normal forms of the respective objects and wrote them into a sorted list; cf. section 3.4). Going over all allowed sublattices for all integer VPMs (for details see section 3.5) we eventually constructed all 4319 reflexive polytopes. The complete list is accessible via internet. ${ }^{1}$ Some statistics is compiled in table 2.

There are several reasons why we decided to store 6202 polytopes rather than 4075 VPMs in the first step of the enumeration process. The fact that polyhedra require less disc space might become important in the context of four dimensional polyhedra. Besides, having the polyhedra explicitly allows us to check for each polyhedron if we already searched the convex subsets of another object that only differs by a lattice automorphism and to avoid the resulting redundancy. The most important advantage is that we could easily check for connectedness: For each new weight system we checked explicitly that at least one of its subpolyhedra had been found before. Connectedness of the original list of 4318 polytopes follows from the fact that this was always the case. All 679 reflexive proper subpolytopes of the exceptional polytope that we only found on a sublattice already show up as subpolyhedra of the 15 maximal objects that live on the original lattices. This establishes connectedness of the complete set of 4319 reflexive polyhedra in 3 dimensions.

An important check for the correct implementation of our classification algorithm is mirror symmetry, i.e. that we obtain for each of the 4319 polyhedra the dual one in the sense of (1). For convenience, we actually checked a slightly weaker statement, namely that we got the dual (i.e., transposed) for each of the 4075 distinct VPMs. The fact, however, that we recovered all 4318 previously found reflexive polyhedra (and, in addition, found the new one) from these VPMs, also provides a very stringent test for the last step of our construction.

In our first complete calculation we stored all 665598 different subpoly-

\footnotetext{
${ }^{1}$ It can be found at http://tph16.tuwien.ac.at $/{ }^{\sim}$ kreuzer $/$ CY.html
} 
topes (on the original lattices) that have an interior point. This took about 2 hours of CPU time and required more than $60 \mathrm{MB}$ of memory. If we only remember the subpolytopes with integer VPM (and thus risk to reanalyze the same non-reflexive objects again and again) computation time triples, but in return we only need less than $1 \mathrm{MB}$ of RAM.

Thinking about the 4-dimensional case, where we have to deal with at least 308 weight systems and with respective point numbers between 47 and 680 , this means, of course, that our program has to be improved drastically. From the above numbers it is clear that we will have to avoid the construction of all IP subpolytopes. In an improved version we terminated the iteration whenever we could verify that all subpolytopes in the present branch would have a facet with distance larger than one from the IP. This reduced the computation time to below 1 minute. With this improved program we already produced about 20 million reflexive 4 -dimensional polytopes and the complete number may well be as large as $10^{9}$, which would surpass the computing resources that presently are at our disposal. Nevertheless, even with an incomplete list, it will be interesting how the spectrum of Hodge numbers of the corresponding Calabi-Yau manifolds changes as compared to the relatively few examples that have been known so far.

Returning to the case of $\mathrm{K} 3$ surfaces, we mention that we also calculated the Picard number for each of our 4319 models, using the formula [7]

$$
\text { Pic }=l\left(\Delta^{*}\right)-4-\sum_{\text {facets } \theta^{*} \text { of } \Delta^{*}} l^{*}\left(\theta^{*}\right)+\sum_{\text {edges } \theta^{*} \text { of } \Delta^{*}} l^{*}\left(\theta^{*}\right) l^{*}(\theta),
$$

where $l$ denotes the number of integer points of a polyhedron and $l^{*}$ denotes the number of interior integer points of a facet or an edge. Contrary to the case of higher dimensional Calabi-Yau manifolds, this number is not the same as the Hodge number $h_{11}$, which is always 20 for K3 surfaces. Instead,

$$
h_{11}=20=\operatorname{Pic}+l(\Delta)-4-\sum_{\text {facets } \theta \text { of } \Delta} l^{*}(\theta),
$$

which is another useful check on our programs. Mirror symmetry for K3 surfaces is usually interpreted in terms of families of lattice polarized K3 surfaces (see, e.g., [24] or [25]). In this context the Picard number of a generic element of a family and the Picard number of a generic element of the mirror family add up to 20 . The fact that the Picard numbers for toric mirror families add up to $20+\sum l^{*}\left(\theta^{*}\right) l^{*}(\theta)$ indicates that our toric models occupy rather special loci in the total moduli spaces.

Let us end this section with briefly discussing a few of the most interesting objects in our list. As table 3 indicates, there are precisely two mirror pairs 
with Picard numbers 1 and 19, respectively. One of them is the quartic hypersurface in $\mathbb{P}^{3}$ with Picard number 1 , together with its mirror of Picard number 19 , which is also the model whose Newton polytope is the only reflexive polytope with only 5 lattice points. This model corresponds to a blow-up of a $\mathbb{Z}^{4} \times \mathbb{Z}^{4}$ orbifold of $\mathbb{P}^{3}$. The blow-up of six fixed lines $z_{i}=z_{j}$ by three divisors each yields 18 exceptional divisors leading to the total Picard number of 19. The other mirror pair with Picard numbers 1 and 19 consists of the hypersurface in $\mathbb{P}_{(1,1,1,3)}^{3}$ of degree 6 and an orbifold of the same model, with Newton polyhedra with 39 and 6 points, respectively. The other polyhedron with the maximal number of 39 points is the Newton polytope of the hypersurface of degree 12 in $\mathbb{P}_{(1,1,4,6)}^{3}$. This model leads to the description of elliptically fibered $\mathrm{K} 3$ surfaces that is commonly used in F-theory applications $[26,27,28]$, with the elliptic fiber embedded in a $\mathbb{P}_{(1,2,3)}^{2}$ by a Weierstrass equation. The mirror family of this class of models can be obtained by forcing two $E_{8}$ singularities into the Weierstrass model and blowing them up. The resulting hypersurface allows also a different fibration structure which can develop an $S O(32)$ singularity; thereby this model is able to describe the F-theory duals of both the $E_{8} \times E_{8}$ and the $S O(32)$ heterotic strings with unbroken gauge groups in 8 dimensions [19].

\section{THE ALGORITHM}

\subsection{GENERAL OUTLINE AND EXISTING RESULTS}

The starting point of our algorithm is the introduction of the concept of a minimal polyhedron [13]. Consider a polyhedron in $\mathbb{R}^{n}$ with the interior point (IP) property. We call this polyhedron minimal if there is no strict subset $\left\{V_{i}, i \in I\right\}, I \subsetneq\{1, \ldots, k\}$ of the set $\left\{V_{1}, \ldots, V_{k}\right\}$ of vertices such that the convex hull of $\left\{V_{i}, i \in I\right\}$ has $\mathbf{0}$ in its interior. It could be shown that these objects allow a classification according to the types of linear relations between its vertices. In particular, in two dimensions only two types are possible: The triangle $V_{1} V_{2} V_{3}$ with

$$
q_{1} V_{1}+q_{2} V_{2}+q_{3} V_{3}=0 \quad \text { where } \quad 0<q_{i}<1, \quad q_{1}+q_{2}+q_{3}=1
$$

and the parallelogram $V_{1} V_{2} V_{1}^{\prime} V_{2}^{\prime}$ with

$$
\begin{gathered}
q_{1} V_{1}+q_{2} V_{2}=0, \quad q_{1}^{\prime} V_{1}^{\prime}+q_{2}^{\prime} V_{2}^{\prime}=0 \quad \text { where } \\
0<q_{i}, q_{i}^{\prime}<1, \quad q_{1}+q_{2}=q_{1}^{\prime}+q_{2}^{\prime}=1 .
\end{gathered}
$$

In a shorthand notation, this result may be summarized as $\{3 ; 2+2\}$. In the same notation the result for three dimensions can be summarized as $\{4 ; 3+2$, 
$\underbrace{3+3} ; 2+2+2\}$ where the underlining symbol indicates a vertex occurring in both linear relations. Here this means that our polyhedron is the convex hull of $V_{1}, V_{2}, V_{3}, V_{2}^{\prime}, V_{3}^{\prime}$ and that there are relations $q_{1} V_{1}+q_{2} V_{2}+q_{3} V_{3}=0$ and $q_{1}^{\prime} V_{1}+q_{2}^{\prime} V_{2}^{\prime}+q_{3}^{\prime} V_{3}^{\prime}=0$. A minimal polyhedron $\nabla_{\min } \subset \mathbb{R}^{n}$ is then specified (up to linear transformations) by its structure and by the weights $q_{i}$ involved in this construction. We will call (combinations of) sets of $q_{i}>0$ with $\sum q_{i}=1$ (combined) weight systems. Notice that everything said so far applies to polyhedra in $\mathbb{R}^{n}$ and real $q_{i}$. In the context of lattice polyhedra (which we will consider henceforth) the $q_{i}$ are rational.

Clearly any polyhedron with the IP property allows at least one (possibly trivial) subset of vertices whose convex hull is a minimal polyhedron. Applying this to the dual $\Delta^{*}$ of a reflexive polyhedron, we find that there exists a minimal integer (not necessarily reflexive) polyhedron $\nabla_{\min } \subseteq \Delta^{*}$, implying $\Delta \subseteq \nabla_{\min }^{*}$. The fact that $\Delta$ is a lattice polyhedron leads to the stronger restriction

$$
\Delta \subseteq \Delta_{\max }:=\text { ConvexHull }\left(\nabla_{\min }^{*} \cap M\right) .
$$

Given a minimal polyhedron $\nabla_{\min } \subset \mathbb{R}^{n}$ we still have to specify a choice of lattice $N \subset N_{\mathbb{R}} \simeq \mathbb{R}^{n}$. The coarsest possible such lattice $N_{\text {coarsest }}$ is the lattice generated by the vertices of $\nabla_{\min }$. Its dual is the finest $M$ lattice $M_{\text {finest }}$. Any other $M$ lattice compatible with integrality of $\nabla_{\min }$ is a sublattice of $M_{\text {finest }}$.

For pairs of reflexive polyhedra clearly only minimal polyhedra and therefore (combined) weight systems such that $\Delta_{\max }$ (w.r.t. $M_{\text {finest }}$ ) has the IP property are relevant. In such a case we also say that a (combined) weight system has the IP property. As a side remark we mention that this definition implies reflexivity of $\Delta_{\max }$ for lattice dimensions $n \leq 4$ [14]. It is easy to see that a combined weight system can have the IP property only if each of its weight systems by itself has it. The weight systems with up to 5 weights with the IP property were classified in [14]. There is one such system $(1 / 2,1 / 2)$ with two weights; with three weights there are the three systems $(1 / 3,1 / 3,1 / 3),(1 / 2,1 / 4,1 / 4),(1 / 2,1 / 3,1 / 6)$; there are 95 systems with four and 184,026 systems with five weights.

Given all simple weight systems, finding the combined weight systems is an easy combinatorial task [23]. From what we have discussed up to now it is clear that any reflexive polyhedron is a subpolyhedron of a maximal polyhedron $\Delta_{\max }$ w.r.t. some (combined) weight system $(q)$ that has the IP property, on some lattice $M$ that is $M_{\text {finest }}$ or a sublattice thereof. Thus the task of classifying reflexive polyhedra is reduced to the task of classifying all reflexive subpolyhedra $\Delta \subset M \subseteq M_{\text {finest }}$ of all maximal polyhedra 
$\Delta_{\max } \subset M_{\text {finest }}$. Before we describe how to find all subpolyhedra of a given polyhedron efficiently, we will show that the set of (combined) weight systems relevant for the classification scheme may still be reduced.

\subsection{NEW DEFINITIONS OF MINIMALITY OF POLYHEDRA}

Remember that our definition of a minimal polyhedron meant that no subset of the set of vertices of $\nabla_{\min }$ should define a polyhedron with the IP property (we might have called such polyhedra vertex-minimal). If we work not just in $\mathbb{R}^{n}$ but with a lattice, we may similarly define a polyhedron $\nabla_{\mathrm{lpm}}$ to be lp-minimal (lp standing for 'lattice point') if no subset of the set of lattice points of $\nabla_{\text {lpm }}$ defines a polyhedron with the interior point property. A (combined) weight system will be called lp-minimal if the corresponding $\nabla_{\min }$ on $N_{\text {coarsest }}$ is lp-minimal. Clearly a polyhedron that is not lp-minimal will contain an lp-minimal polyhedron as a proper subset; therefore only lp-minimal polyhedra will play a role in our classification scheme.

Note, however, that even being lp-minimal does not guarantee that $\Delta_{\max }$ is not a subpolyhedron of any other reflexive polyhedron: $\Delta_{\max }^{*} \underset{\tilde{\nabla}}{\operatorname{might}}$ contain not only $\nabla_{\text {lpm }}$, but also a different lp-minimal polyhedron $\tilde{\nabla}_{\text {lpm }}$ such that $\Delta_{\max } \subseteq \tilde{\Delta}_{\max }$. This cannot happen, however, if it is impossible to omit any of the vertices of $\nabla_{\min }$ from $\Delta_{\max }^{*} \cap N$ without violating the IP property. In that case we call the corresponding (combined) weight systems very minimal. Clearly very minimal implies lp-minimal.

As an example of a polyhedron that is lp-minimal but not very minimal consider $\nabla_{\min }$ defined by the single weight system $(1,2,3,5) / 11$. Here we may represent the vertices of $\nabla_{\min }$ by

$$
V_{1}=(1,0,0), V_{2}=(0,1,0), V_{3}=(0,0,1) \text { and } V_{4}=(-2,-3,-5),
$$

the only other lattice point being $\mathbf{0}$. As $\nabla_{\min }^{*}$ is not a lattice polyhedron, $\Delta_{\max }$ is smaller than $\nabla_{\min }^{*}$ and so $\Delta_{\max }^{*}$ is larger than $\nabla_{\min }$. More precisely, $\Delta_{\max }^{*}$ has the three additional vertices

$$
V_{5}=(-2,-3,-6), V_{6}=(0,-1,-1) \text { and } V_{7}=(-1,-1,-2)
$$

and besides contains 6 further lattice points. Thus $\nabla_{\min }^{(1,2,3,5) / 11}$ contains $\nabla_{\min }^{(1,2,3,6) / 12}$ (the convex hull of $\left.V_{1}, V_{2}, V_{3}, V_{5}\right)$ and $\nabla_{\min }^{(1,1,1,2) / 5}$ (the convex hull of $\left.V_{1}, V_{2}, V_{3}, V_{7}\right)$. In addition it contains some other minimal polyhedra corresponding to other lattice points. Therefore $\Delta_{\max }^{(1,2,3,5) / 11}$ is contained 
in other maximal polyhedra and does not play a role in the classification algorithm.

An example of a very minimal weight system is $(1,1,3,4) / 9$. The vertices of $\nabla_{\min }$ can be represented as

$$
V_{1}=(1,0,0), V_{2}=(0,1,0), V_{3}=(0,0,1) \text { and } V_{4}=(-1,-3,-4),
$$

the only other lattice point again being $\mathbf{0} . \Delta_{\max }^{*}$ has the additional points

$$
V_{5}=(0,-2,-3), P_{6}=(0,-1,-2), P_{7}=(0,0,-1) \text { and } P_{8}=(0,0,-1)
$$

( $V_{5}$ is the only vertex among them). Dropping any of the vertices $V_{1}, \ldots, V_{4}$ from $\Delta_{\max }^{*}$ results in loss of the IP property, as is easily checked. Thus there cannot be a weight system $(q) \neq(1,1,3,4) / 9$ such that $\left(\Delta_{\max }^{(q)}\right)^{*} \subseteq$ $\left(\Delta_{\max }^{(1,1,3,4) / 9}\right)^{*}$ or, conversely, that $\Delta_{\max }^{(1,1,3,4) / 9} \subseteq \Delta_{\max }^{(q)}$.

Let us summarize: By the analysis given in this subsection, every reflexive polyhedron is contained in the dual of an lp-minimal polyhedron, and the duals of very minimal polyhedra are not contained in the duals of any other minimal polyhedra.

Our computer programs led to the following further statements: For $n=3$, there are 15 very minimal weight systems and 4 further weight systems that are lp-minimal without being very minimal. The latter weight systems lead to polytopes $\Delta_{\max }$, however, that are contained in the $\Delta_{\max }$ coming from the 15 very minimal weight systems.

\subsection{FINDING SUBPOLYHEDRA}

Finding all subpolyhedra of a given polyhedron without introducing excessive redundancy is a non-trivial task. Postponing the problem of identifying polyhedra that are related by lattice automorphisms to the next section, the first step is to construct all convex subsets of lattice points. This can be achieved by first generalizing the problem: Consider the task of finding all subpolyhedra of a given polyhedron $\Delta$ that contain a specific subset of the vertices of $\Delta$ (let us call these allowed subpolyhedra). We may think of specifying this subset by attaching labels $k$ (for 'keep') to these vertices. Finding the corresponding subpolyhedra of $\Delta$ can be done recursively: Unless $\Delta$ is empty or all of the vertices of $\Delta$ carry $k$ labels, we may choose a vertex $V$ that does not carry a $k$ label. Then the allowed subpolyhedra of $\Delta$ will be the allowed subpolyhedra of $\Delta \backslash\{V\}$ and the allowed subpolyhedra of $\Delta$ with an extra $k$ label attached to $V$. Clearly the recursion terminates only 
when $\Delta$ is empty (or, in our case we let it terminate whenever it ceases to have the interior point property) or when all of its vertices carry $k$ labels.

Our original problem can thus be solved with the following recursive algorithm:

(1) Start with $\Delta_{\max }$ without labels

(2) At a specific step in the recursion we have a polyhedron $\Delta$, some of whose vertices carry $k$ labels. If $\Delta$ doesn't have the IP property, we don't continue with it. Otherwise, if all vertices of $\Delta$ have $k$ labels, we can add $\Delta$ to the list of subpolyhedra of $\Delta_{\max }$. Otherwise, pick a specific vertex $V$ without a label. Do step (2) with the label of $V$ changed to $k$ and with $\Delta^{\prime}=\Delta \backslash\{V\}$.

As a vertex of a polyhedron is also a vertex of any subpolyhedron to which it belongs, a point that carries a $k$ label is always guaranteed to be a vertex.

The application of this algorithm to the Newton polygon of $\mathbb{P}_{(1,2,3)}$ is shown pictorially in figure 1 . Note how every subpolyhedron occurs precisely once as an endpoint of the recursive tree.

\subsection{NORMAL FORMS OF POLYHEDRA}

In a classification scheme like the present one that produces the complete set with large redundancy it is useful to define normal forms of the relevant objects. A normal form allows us to define a total ordering (for example lexicographic) so that we can efficiently check for new entries by searching a sorted list with bisection.

To describe our polyhedra we start with the matrix of coordinates of the vertices. This matrix is determined only up to an $S_{n_{V}} \times G L(d, \mathbb{Z})$ symmetry, where $S_{n_{V}}$ is the group of permutations of the vertices and $G L(d, \mathbb{Z})$ is the group of coordinate transformations of a $d$-dimensional lattice. To lift this redundancy we first define a normal form for vertex pairing matrices using permutations of lines and columns to obtain the (lexicographically) maximal matrix. This removes the $S_{n_{V}}$ degeneracy, except for the subgroup that corresponds to the symmetry of the polyhedron on the coarsest lattice, if we demand that the ordering of the vertices should be the same as in the normal form of the vertex pairing matrix. Given a particular ordering of the vertices we then use the $G L(d, \mathbb{Z})$ freedom to make the matrix of vertices upper diagonal with positive elements along the diagonal and minimal nonnegative elements above. 


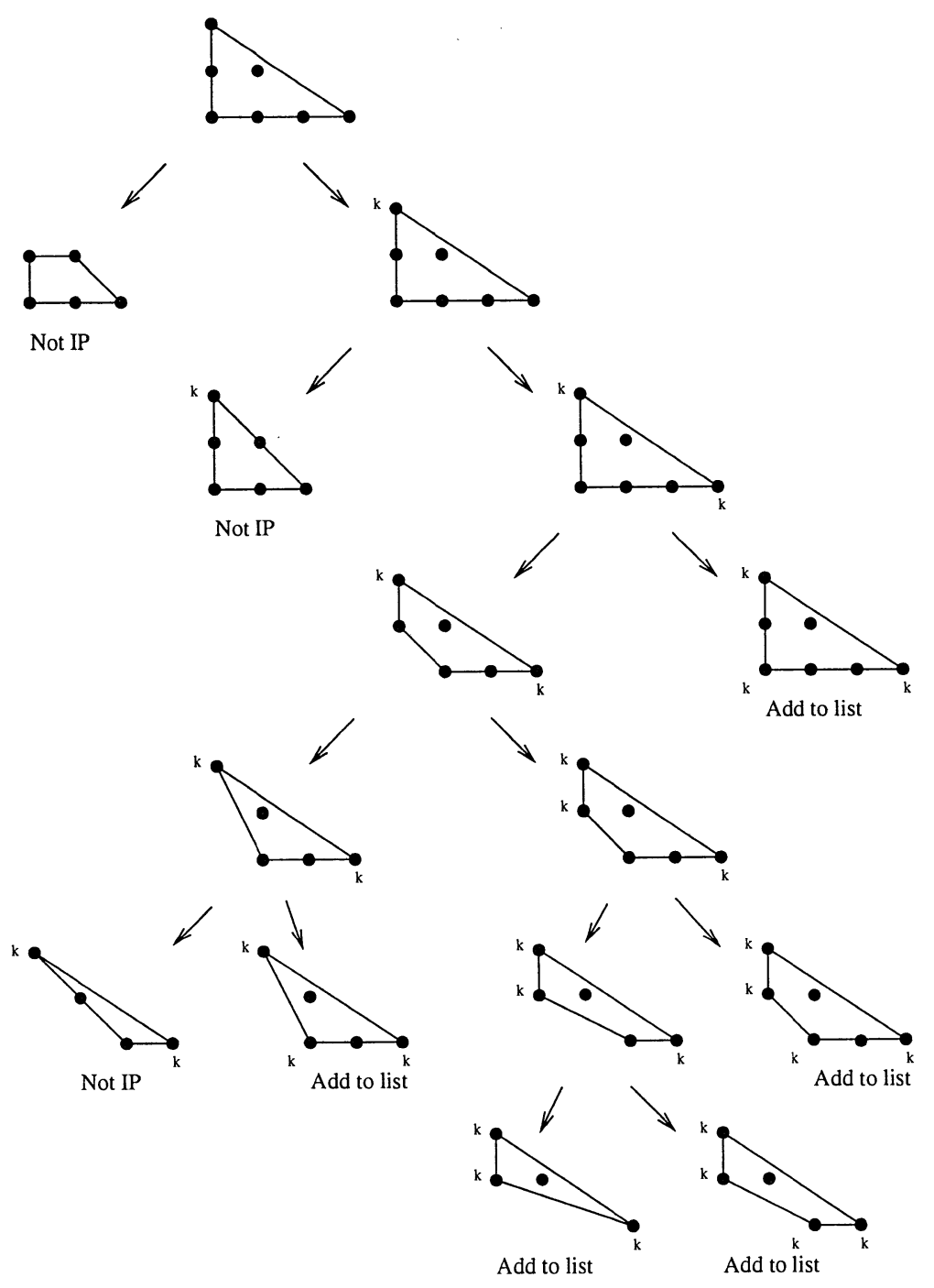

Figure 1: Applying the classification algorithm to $\Delta\left(\mathbb{P}_{(1,2,3)}\right)$

In the cases where the normal form for vertex pairing matrices has not determined the ordering of vertices unambiguously, we do this for every allowed ordering and choose the lexicographically smallest one to be our normal form.

Invariances of this normal form correspond to symmetries of the polyhedron.

To make sure that we cannot miss any reflexive polyhedron in our classification scheme we have to keep record of any polyhedron that is reflexive either on the lattice on which it was found or on any sublattice. A necessary 
and sufficient condition for a polyhedron to be reflexive on some lattice is just integrality of the vertex pairing matrix: If $\Delta$ is reflexive, then its vertex pairing matrix will obviously be integer, and if the vertex pairing matrix is integer, $\Delta$ will be reflexive on the lattice generated by the vertices of $\Delta$. So the minimal set of polyhedra to be stored is the set of those with integer vertex pairing matrix. For saving calculation time, however, it makes sense to keep record of non-reflexive polyhedra as well, thereby increasing the required storage capacity. For the present case of classifying three-dimensional reflexive polyhedra it doesn't really matter if one chooses to keep record of non-reflexive polyhedra. It seems, however, that for four-dimensional polyhedra memory seems to be a greater problem than time, so it probably never makes sense to keep record of polyhedra that are not reflexive on any lattice.

\subsection{SUBLATTICES}

Given an integer vertex pairing matrix, there is always a coarsest lattice on which the polyhedron $\Delta$ is reflexive (the lattice generated by the vertices of $\Delta$ ) and a finest lattice (the lattice dual to the one generated by the vertices of $\Delta^{*}$ ). The quotient of these lattices is a finite Abelian group. This group can be represented by expressing the generators of the finer lattice in terms of the generators of the coarser lattice. The corresponding vectors in $\mathbb{Q}^{n}$, taken modulo $\mathbb{Z}^{n}$, are the generators of the quotient group.

A vertex pairing matrix $X$ is an $n_{F} \times n_{V}$ matrix, $n_{F}$ and $n_{V}$ being the numbers of facets (dual vertices) and vertices, respectively. $X$ can be decomposed $X=W \cdot D \cdot U$ where $W$ is $n_{F} \times d, D$ is a $d \times d$ diagonal matrix and $U$ is $d \times n_{V}$. Before giving a geometrical interpretation, let us see how this decomposition can be achieved algorithmically: By recombining the lines and columns of $X$ in the style of Gauss's algorithm for solving systems of linear equations, we can turn $X$ into an $n_{F} \times n_{V}$ matrix $\tilde{D}$ with non-vanishing elements only along the diagonal. But recombining lines just corresponds to left multiplication with some $G L(\mathbb{Z})$ matrix, whereas recombining columns corresponds to right multiplication with some $G L(\mathbb{Z})$ matrix. Keeping track of the inverses of these matrices, we successively create decompositions $X=\tilde{W}^{(n)} \cdot \tilde{D}^{(n)} \cdot \tilde{U}^{(n)}\left(\right.$ with $\tilde{W}^{(0)}=1, \tilde{D}^{(0)}=X$ and $\tilde{U}^{(0)}=1$ ). Let us denote the matrices resulting from the last step by $\tilde{W}$, $\tilde{D}$ and $\tilde{U}$. $\tilde{W}$ and $\tilde{U}$ being regular matrices and the rank of $X$ being $d$, it is clear that $\tilde{D}$ has only $d$ non-vanishing elements which can be taken to be the first $d$ diagonal elements. Then we can choose $W$ to consist of the first $d$ columns of $\tilde{W}, U$ to consist of the first $d$ lines of $\tilde{U}$ and $D$ to be the upper left $d \times d$ block of $\tilde{D}$. 
The interpretation of $U$ and $W$ is as follows: We may view the columns of $X$ as the coordinates of $\Delta$ (on $M_{\text {finest }}$ ) in an auxiliary $n_{F}$-dimensional space carrying an $n_{F}$-dimensional lattice in which $\Delta$ is embedded. The $n_{F} \times n_{F}$ matrix $\tilde{W}$ effects a change of coordinates in the $n_{F}$-dimensional lattice so that $\Delta$ now lies in the lattice spanned by the first $d$ coordinates. Thus we can interpret the columns of $D \cdot U$ as the vertices of $\Delta$ on $M_{\text {finest }}$. Similarly, the lines of $W \cdot D$ are coordinates of the vertices of $\Delta^{*}$ on $N_{\text {finest }}$, whereas $U$ and $W$ are the corresponding coordinates on the coarsest possible lattices.

More explicitly, denoting the generators of $M_{\text {coarsest }}$ by $\vec{E}_{i}$ and the generators of $M_{\text {finest }}$ by $\vec{e}_{i}$, we have $\vec{E}_{i}=\vec{e}_{j} D_{j i}$. An intermediate lattice will have generators $\overrightarrow{\mathcal{E}}_{i}=\vec{e}_{j} T_{j i}$ such that the $\vec{E}_{i}$ can be expressed in terms of the $\overrightarrow{\mathcal{E}}_{j}$, amounting to

$$
\vec{E}_{i}=\overrightarrow{\mathcal{E}}_{j} S_{j i}=\vec{e}_{k} T_{k j} S_{j i}
$$

with some integer matrix $S$. This results in the condition $D_{k i}=T_{k j} S_{j i}$. In order to get rid of the redundancy coming from the fact that the intermediate lattices can be described by different sets of generators, one may proceed in the following way: $\overrightarrow{\mathcal{E}}_{1}$ may be chosen as a multiple of $\vec{e}_{1}$ (i.e., $\overrightarrow{\mathcal{E}}_{1}=\vec{e}_{1} T_{11}$ ). Then we choose $\overrightarrow{\mathcal{E}_{2}}$ as a vector in the $\vec{e}_{1}-\vec{e}_{2}$-plane (i.e., $\overrightarrow{\mathcal{E}}_{1}=\vec{e}_{1} T_{12}+\vec{e}_{2} T_{22}$ ) subject to the condition that the lattice generated by $\overrightarrow{\mathcal{E}}_{1}$ and $\overrightarrow{\mathcal{E}}_{2}$ should be a sublattice of the one generated by $\vec{E}_{1}$ and $\vec{E}_{2}$, which is equivalent to the possibility of solving $T_{k j} S_{j i}=D_{k i}$ for integer matrix elements of $S$. We may avoid the ambiguity arising by the possibility of adding a multiple of $\mathcal{E}_{1}$ to $\mathcal{E}_{2}$ by demanding $0 \leq T_{12}<T_{11}$. We can choose the elements of $T$ column by column (in rising order). For each particular column $i$ we first pick $T_{i i}$ such that it divides $D_{i i}$; then $S_{i i}=D_{i i} / T_{i i}$. Then we pick the $T_{j i}$ with $j$ decreasing from $i-1$ to 1 . At each step the $j$ 'th line of $T \cdot S=D$,

$$
T_{j i} S_{i i}+\sum_{j<k<i} T_{j k} S_{k i}+T_{j j} S_{j i}=0,
$$

must be solved for the unknown $T_{j i}$ and $S_{j i}$ with the extra condition $0 \leq$ $T_{j i}<T_{i i}$ ensuring that we get only one representative of each equivalence class of bases. Proceeding in this way, we create all inequivalent upper triangular matrices $T$ and $S$ such that the coordinates of the vertices of $\Delta^{*}$ on the intermediate lattice given by the $\overrightarrow{\mathcal{E}}_{i}$ are the columns of $S \cdot U$ and the vertices of $\Delta^{*}$ on the corresponding dual lattice are $W \cdot T$. This completes our discussion of the building blocks that we needed to implement our construction.

Acknowledgements. This work is supported in part by the Austrian Research Funds FWF (Schrödinger fellowship J1530-TPH), NSF grant PHY9511632, the Robert A. Welch Foundation and by the Austrian National Bank under grant Nr. 6632 . 


\section{References}

[1] P. Candelas, A.T. Horowitz, A. Strominger, E. Witten, Vacuum Configurations for Superstrings, Nucl. Phys. B258 (1985) 46.

[2] P. Candelas, M. Lynker and R. Schimmrigk, Calabi-Yau Manifolds in Weighted $\mathbb{P}_{4}$, Nucl. Phys. B341 (1990) 383.

[3] C.Vafa, String vacua and orbifoldized LG models, Mod. Phys. Lett. A4 (1989) 1169 ;

Superstring vacua, HUTP-89/A057 preprint

[4] P. Berglund, T. Hübsch, A Generalized Construction of Mirror Manifolds, Nucl. Phys. B393 (1993) 377, hep-th/9201014.

[5] A. Klemm, R. Schimmrigk, Landau-Ginzburg String Vacua, Nucl. Phys. B411 (1994) 559, hep-th/9204060.

[6] M. Kreuzer, H. Skarke, No Mirror Symmetry in Landau-Ginzburg Spectra!, Nucl. Phys. B388 (1992) 113, hep-th/9205004.

[7] V.V. Batyrev, Dual Polyhedra and Mirror Symmetry for Calabi-Yau Hypersurfaces in Toric Varieties, J. Alg. Geom. 3 (1994) 493, alg-geom/9310003.

[8] M. Reid, The moduli space of 3-folds with $K=0$ may nevertheless be irreducible, Math. Ann. 278 (1987) 329.

[9] A. Strominger, Massless Black Holes and Conifolds in String Theory, Nucl. Phys. B451 (1995) 96, hep-th/9504090.

[10] B.R. Greene, D.R. Morrison, A. Strominger, Black Hole Condensation and the Unification of String Vacua, Nucl. Phys. B451 (1995) 109, hep-th/9504145.

[11] T.-M. Chiang, B.R. Greene, M. Gross, Y. Kanter, Black Hole Condensation and the Web of Calabi-Yau Manifolds, hep-th/9511204.

[12] A.C. Avram, P. Candelas, D. Jancic, M. Mandelberg, On the Connectedness of the Moduli Space of Calabi-Yau Manifolds, Nucl. Phys. B465 (1996) 458, hep-th/9511230.

[13] M. Kreuzer, H. Skarke, On the Classification of Reflexive Polyhedra, Commun. Math. Phys. 185 (1997) 495, hep-th/9512204.

[14] H. Skarke, Weight Systems for Toric Calabi-Yau Varieties and Reflexivity of Newton Polyhedra, Mod. Phys. Lett. A11 (1996) 1637, alg-geom/9603007.

[15] P. Candelas and A. Font, Duality Between the Webs of Heterotic and Type II Vacua, hep-th/9603170, revised version (march 1997).

[16] A. Avram, M. Kreuzer, M. Mandelberg, H. Skarke, Searching for K3 Fibrations, Nucl. Phys. B494 (1997) 567, hep-th/9610154.

[17] M. Kreuzer, H. Skarke, Calabi-Yau Fourfolds and Toric Fibrations, J. Geom. Phys. 466 (1997) 1, hep-th/9701175.

[18] E. Perevalov, H. Skarke, Enhanced Gauge Symmetry in Type II and F-Theory Compactifications: Dynkin Diagrams from Polyhedra, Nucl. Phys. B505 (1997) 679, hep-th/9704129.

[19] P. Candelas, H. Skarke, F-theory, SO(32) and Toric Geometry, Phys. Lett. B413 (1997) 63, hep-th/9706226.

[20] G.M.Ziegler, Lectures on polytopes, GTM 152 (Springer, Berlin 1995)

[21] W. Fulton, Introduction to Toric Varieties (Princeton Univ. Press, Princeton 1993). 
[22] T. Oda, Convex Bodies and Algebraic Geometry (Springer, Berlin Heidelberg 1988).

[23] A. Avram, M. Kreuzer, M. Mandelberg, H. Skarke, The Web of Calabi-Yau Hypersurfaces in Toric Varieties, Nucl. Phys. B505 (1997) 625, hep-th/9703003.

[24] Igor V. Dolgachev, Mirror symmetry for lattice polarized K3 surfaces, alggeom/9502005.

[25] P.S. Aspinwall, K3 Surfaces and String Duality, hep-th/9611137.

[26] C. Vafa, Evidence for F-Theory, Nucl. Phys. B469 (1996) 403, hepth/9602022.

[27] D.R. Morrison and C. Vafa, Compactifications of F-Theory on Calabi-Yau Threefolds - I, Nucl. Phys. B473 (1996) 74, hep-th/9602114.

[28] D.R. Morrison and C. Vafa, Compactifications of F-Theory on Calabi-Yau Threefolds - II, Nucl. Phys. B476 (1996) 437, hep-th/9603161. 